admission creatinine, $\mathrm{pH}$, worst arterial carbon dioxide level $\left(\mathrm{CO}_{2}\right)$ and presence and severity of chronic kidney disease (CKD) was assessed by simple linear regression.

Results There was a statistically significant regression coefficient between worst observed $\mathrm{CO}_{2}$ and the duration of NIV (fitted equation: NIV Duration $=4.281+14.357 \times$ Worst $\mathrm{CO}_{2}, \mathrm{p}=$ $0.019)$. The plotted linear relationship showed an increase in duration of NIV treatment of $14.35 \mathrm{~h}$ for every $1 \mathrm{kPa}$ increase in $\mathrm{CO}_{2}$ above $6 \mathrm{kPa}$. The admission creatinine and severity of CKD did not significantly alter the duration of NIV required. The presence of acute kidney injury was also not significant. The $\mathrm{pH}$ value did not significantly alter the duration of NIV treatment.

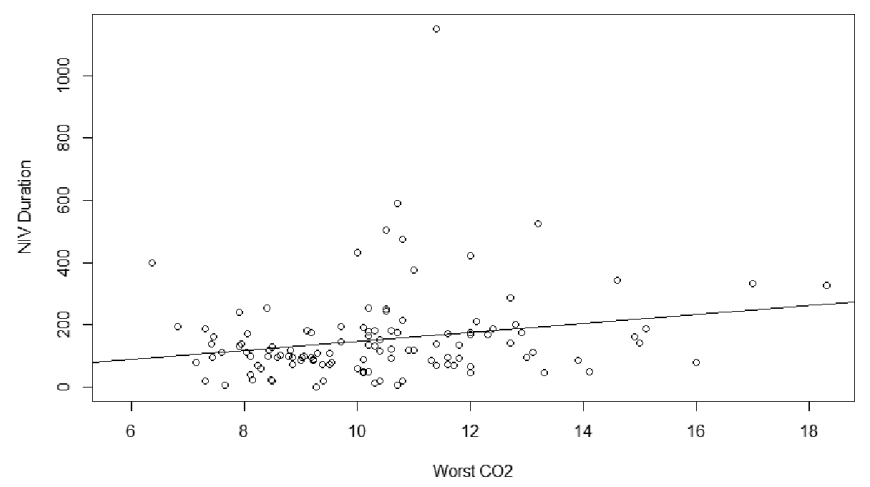

Abstract P42 Figure 1 NIV duration plotted against worst $\mathrm{CO}_{2}$. Linear regression fitted equation shown (duration NIV (hours) $=4.281$ $+14.357 \times$ Worst $\mathrm{CO}_{2}$ )

Conclusion This survey shows that the level of $\mathrm{CO}_{2}$ influences the duration of acute NIV required, in that for every $1 \mathrm{kPa}$ rise in $\mathrm{CO}_{2}$, the duration of acute NIV treatment rises by $14.35 \mathrm{~h}$. The other studied variables do not correlate with treatment duration.

\section{P43 HOW APPROPRIATELY IS NIV USED AS A CEILING OF TREATMENT?}

S Woolf, I Jitan, J Robinson, I Suntharalingam. Royal United Hospitals NHS Foundation Trust, Bath, UK

10.1136/thoraxjnl-2015-207770.180

Background Treatment Escalation Plans (TEPs) were introduced at the Royal United Hospitals Bath (RUH) in 2013 to help physicians document decisions regarding ceilings of treatment. In implementing a TEP, a patient may be deemed unsuitable for resuscitation and/or Intensive Care Unit (ICU) but remain a potential candidate for ward-based non-invasive ventilation (NIV). However as ward-based NIV is indicated in relatively few respiratory conditions this option should only be available to a small cohort of patients. This study examines how appropriately patients have NIV cited as a ceiling of treatment, using 2002 BTS acute NIV guidelines as a benchmark.

Method We collected data from medical, surgical and geriatric wards at the RUH on three separate days between November 2014 and June 2015. In patients with a TEP who were deemed unsuitable for CPR, we recorded a) the ceiling of treatment decision b) reason for admission and c) co-morbidities. We reviewed how many patients with NIV as a ceiling of treatment had an indication in accordance with BTS guidelines.
Results 658 patient notes were reviewed. 109/658 patients were deemed not suitable for ICU but had NIV as a ceiling of treatment. 64/109 patients (59\%) had an indication in accordance with BTS guidelines, while 45/109 patients (41\%) were noncompliant. There was variation in compliance between specialties (General Medicine 60\% compliant, Elderly Care 54\% compliant and Surgery 33\% compliant). The Respiratory ward was the most compliant (100\%).

Conclusions Whilst NIV can offer significant survival benefits to patients with certain conditions (eg COPD exacerbations, obesity hypoventilation syndrome and chest wall disease) national BTS audits have repeatedly shown that ward-based NIV is often used unsuccessfully outside of these indications. The current study demonstrates that over $40 \%$ of patients admitted to our hospital inappropriately have NIV set as their ceiling of treatment, albeit with some variability between wards and specialties. This suggests that further education is required about the potential limitations of NIV, particularly for non-respiratory specialists who often make TEP decisions.

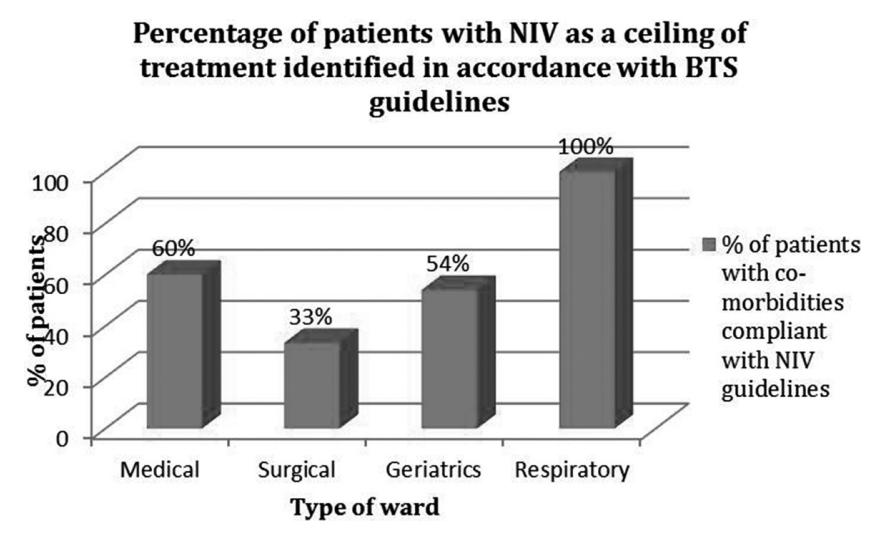

Abstract P43 Figure 1

\section{CHRONIC OBSTRUCTIVE PULMONARY DISEASE EXACERBATION AND RESPIRATORY ACIDOSIS: PATIENT OUTCOMES AT 6 MONTHS}

${ }^{1} \mathrm{~S}$ Jackson, ${ }^{2} \mathrm{TM}$ McKeever, ${ }^{3} \mathrm{G}$ Hearson, ${ }^{4} \mathrm{G}$ Housley, ${ }^{3} \mathrm{C}$ Reynolds, ${ }^{1} \mathrm{~W}$ Kinnear, ${ }^{3}$ TW Harrison, ${ }^{5}$ AM Kelly, ${ }^{3}$ DE Shaw. ${ }^{1}$ Nottingham University Hospitals NHS Trust, Nottingham, Nottinghamshire; 'Division of Epidemiology, University of Nottingham, Nottingham, UK; ${ }^{3}$ Respiratory Research Unit, Division of Respiratory Medicine, University of Nottingham, Nottingham, UK; ${ }^{4}$ East Midlands Academic Health Sciences Network, Nottingham, UK; ${ }^{5}$ Joseph Epstein Centre for Emergency Medicine Research, Western Health, St Albans, Victoria, Australia

\subsection{6/thoraxjn|-2015-207770.181}

Introduction Recognition of hypercapnic respiratory failure is a vital part of the assessment and management of the patient with an acute exacerbation of chronic obstructive pulmonary disease (COPD). Several studies have demonstrated that respiratory acidosis in the context of an acute exacerbation is associated with worse inpatient outcomes. Our study compares the outcomes of patients admitted with an acute exacerbation, between those with respiratory acidosis and those who had a normal $\mathrm{pH}$ and $\mathrm{PaCO}_{2}$ on arterial blood gas (ABG) analysis.

Methods Patients requiring hospital treatment for an acute exacerbation of COPD had an ABG taken on admission. Patients were subsequently assessed for the following outcomes: inpatient 
mortality, outpatient mortality up to six months after discharge and hospital re-admission rates in the six months post discharge. Chi-squared test was applied to assess the relationship between respiratory acidosis and our outcomes.

Results 234 patients had an admission ABG and were subsequently followed up to the point of death or six months post discharge. Patients with a $\mathrm{PaCO}_{2}$ of $>6 \mathrm{Kpa}$ were 2.33 times (95\% CI 1.11 to 4.96 ) more likely to die in hospital as compared to those patients with a normal value. Patients with a lower arterial $\mathrm{pH}(<7.35)$ were 2.32 times $(95 \%$ CI 1.07 to 4.96) more likely to die in hospital as compared to those with a $\mathrm{pH}$ of $>7.35$. The increased risk in mortality was only seen for in-hospital mortality and there was no association with death in the 6 months following discharge, hospital re-admission or readmission for a respiratory problem.

Conclusion This data supports previous studies that suggest hypercapnia and respiratory acidosis are associated with increased inpatient mortality, therefore further demonstrating the usefulness of $\mathrm{pH}$ and $\mathrm{PaCO}_{2}$ as prognostic markers for inpatient outcomes. However our study does suggest that patients with respiratory acidosis on admission, who survive until discharge from hospital, do not have an increased risk of six month mortality or readmission compared to those with a normal admission ABG.

\section{P45 PRACTICAL USE OF THE DECAF SCORE: CAN WE IMPROVE OUTCOMES IN ACUTE EXACERBATION OF COPD ADMISSIONS?}

L Collier, T David, C Craig, R Yadavilli. Royal Bolton Hospital, Bolton, UK

\subsection{6/thoraxjnl-2015-207770.182}

Background Acute exacerbations of COPD (AECOPD) are the second most common cause of emergency hospital admission in England and are associated with an inpatient mortality rate of 4.3\%. ${ }^{1}$ The Dyspnoea, Eosinopenia, Consolidation, Acidaemia and Atrial Fibrillation (DECAF) Score, is an effective prognostic tool that predict mortality in AECOPD admissions. This scoring system is easy to apply during admission and has performed better than existing prognostic tools. ${ }^{2}$ We aim to appraise the efficacy of DECAF score in our busy respiratory and medical admissions unit.

Method Hospital admissions with AECOPD from Dec 2014 to Mar 2015 are prospectively reviewed and DECAF score applied to each patient. Morbidity and mortality indicators were then correlated with both total DECAF scores and each predictive index.

Results 78 admissions were reviewed, 60\% were male and the mean age was 72.7 years. Average length of stay was 15.3 days and 12 patients died in hospital. Our results were comparable with previous studies ${ }^{3}$, with inpatient mortality highest in those with DECAF scores of 3-5 (92\%) and lowest in those with scores of $0-1(0 \%)$. Higher DECAF scores were also associated with use of non-invasive ventilation (43\%).

Furthermore, each individual predictive index within the DECAF score was independently related to an increased mortality rate. There was $44 \%$ mortality in patients with atrial fibrillation and $30 \%$ mortality in patients with dyspnoea score of eMRC 5B. In-hospital mortality rate increased with each DECAF score (Figure 1).

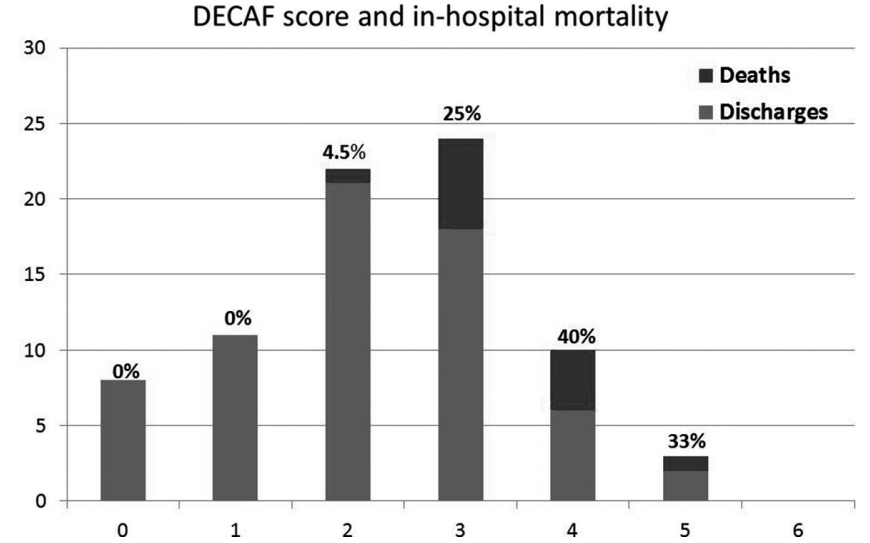

Abstract P45 Figure 1

Conclusions Introduction of DECAF score as clinical prediction tool for AECOPD admissions in our departments may be beneficial in reducing morbidity and mortality. Those scoring highest should be considered for early escalation, higher level of care and or palliative management. Those with lower scores may be suitable for early supported discharge. Further study of a larger group however is advisable to confirm the significance of these findings.

\section{REFERENCES}

1 National Chronic Obstructive Pulmonary Disease Audit Programme: Clinical audit of COPD exacerbations admitted to acute units in England and Wales, 2014

2 J Steer, J Gibson, S Bourke. The DECAF Score: predicting hospital mortality in exacerbations of chronic obstructive pulmonary disease. Thorax 2012;67:970-976

\section{P46 FREQUENCY OF COPD EXACERBATIONS IN THE GERMAN DACCORD REGISTRY}

${ }^{1} \mathrm{P}$ Kardos, ${ }^{2} \mathrm{R}$ Buhl, ${ }^{3} \mathrm{C}-\mathrm{P}$ Criée, ${ }^{4} \mathrm{C}$ Vogelmeier, ${ }^{5} \mathrm{C}$ Mailaender, ${ }^{6} \mathrm{H}$ Worth. ${ }^{1}$ Group Practice and Centre for Allergy, Respiratory and Sleep Medicine, Red Cross Maingau Hospita, Frankfurt, Germany; '2Pulmonary Department, Mainz University Hospital, Frankfurt, Germany; ${ }^{3}$ Department of Sleep and Respiratory Medicine, Evangelical Hospital GoettingenWeende, Bovenden, Germany: ${ }^{4}$ Department of Respiratory Medicine, University of Marburg, Marburg, Germany; ${ }^{5}$ Novartis Pharma GmbH, Nuremberg, Germany; ${ }^{6}$ Facharztforum Fuerth, Fuerth, Germany

\subsection{6/thoraxjnl-2015-207770.183}

Introduction In patients with COPD, exacerbations are among the most relevant safety measures. In this analysis of data from the observational DACCORD study, we report the frequency of exacerbations in a COPD population.

Methods To get insights into occurrence and frequency of exacerbations, data from 4,123 patients were obtained from 349 primary and secondary care centres in Germany. To be eligible for entry into DACCORD, all patients had to have a COPD diagnosis (consistent with the German Disease Management Programme definition), and had to have a change in bronchodilator maintenance medication, prior to entry. Data collected included history and treatment of exacerbations 6 months prior to inclusion, and for the duration of follow-up. Exacerbations were defined based on prescription of oral corticosteroids and/or antibiotics or on hospitalisation.

Results Mean age of the patients was 65.7 years; 36.9\% of patients had severe or very severe airflow limitation (GOLD $2010)$. In the 6 month period prior to study inclusion, $26.4 \%$ of the patients had at least one exacerbation. Fewer patients in the subgroup with CAT30 (16.7\% vs $47.9 \%$ ). Interestingly, $45 \%$ of 